\title{
The role of adrenomedullin and galanin in recurrent vasovagal syncope: a case control study
}

\author{
Jiri Plasek', Vlastimil Doupal ${ }^{b}$, Jana Furstovac, Tomas Furst ${ }^{d}$, Kristian Safarcike, Alena Krnacova ${ }^{f}$, Nadezda Petejovaa, \\ Zuzana Hrabovska ${ }^{a}$, Arnost Martinek ${ }^{\mathrm{a}}$, Milos Taborsky ${ }^{\mathrm{b}}$
}

\begin{abstract}
Aims. Orthostatic stimuli are known to elicit changes in vasoactive peptide levels. The hypothesis of no difference in adrenomedullin and/or galanin levels in patients with recurrent vasovagal syncope and healthy controls was tested in a passive 35 -min head-up tilt test (HUTT).

Methods. Twenty eight persons ( 14 patients and 14 healthy controls) were tested in a $35-\mathrm{min} / 60^{\circ}$ head-up tilt test with telemetry monitoring. Three blood samples were evaluated for each person during the HUTT. Plasma levels of adrenomedullin and galanin were analysed by the Kruskal-Wallis test for all sampling periods. Vagal influence was indirectly assessed by the break index.

Results. There were no significant differences between groups in median values for either adrenomedullin or galanin plasma levels (all $6 \mathrm{p}$-values were greater than 0.4). For adrenomedullin, no significant difference between groups was found. For galanin, the rate of change between the 1st and 2nd measurement was significantly greater for patients $(P=0.04)$, regardless of HUTT result but between the $2^{\text {nd }}$ and $3^{\text {rd }}$ measurement it was insignificant $(P=0.36)$. In the group of positive cases, the break index increased significantly $(P=0.02)$.

Conclusion. We confirmed that there is a different galanin secretion pattern during orthostatic provocation in patients with recurrent vasovagal syncope than healthy individuals. For adrenomedullin, no significant difference was found. A significant increment of the break index confirmed increased vagal influence in the subgroup of positive cases.
\end{abstract}

Key words: adrenomedullin, galanin, vasovagal syncope, head-up tilt test

Received: July 12, 2011; Accepted with revision: November 10, 2011; Available online: December 20, 2011 http:/dx.doi.org/10.5507/bp.2011.061

${ }^{a}$ Clinic of Internal Medicine, University Hospital Ostrava and Faculty of Medicine, University of Ostrava, Czech Republic ${ }^{b}$ Department of Internal Medicine I - Cardiology, University Hospital Olomouc and Faculty of Medicine and Dentistry, Palacky University Olomouc

'First Faculty of Medicine, Charles University Prague

${ }^{d}$ Department of Mathematical Analysis and Applications of Mathematics, Faculty of Science, Palacky University Olomouc

${ }^{e}$ Center of Laboratory Diagnostics, University Hospital Ostrava

${ }^{f}$ Department of Clinical Biochemistry, University Hospital Ostrava

Corresponding author: Milos Taborsky, e-mail: milos.taborsky@fnol.cz

\section{INTRODUCTION}

According to the European Society of Cardiology Guidelines ${ }^{1}$ syncope is a symptom defined as a transient, self-limited loss of consciousness and postural tone. The onset of syncope is relatively rapid, and the subsequent recovery is spontaneous, complete, and usually prompt. Vasovagal syncope (VVS) is accompanied by inactivation of sympathetic tone, and paradoxical activation of vagal tone leading to vasodepressor, cardio-inhibitory or mixed reaction. Some vasoactive peptides are reported to be involved in the reaction to orthostatic stress but their particular roles and interactions are complex and poorly understood ${ }^{2}$. In this study, adrenomedullin and galanin were selected and their role in the vasovagal response was assessed. Both peptides are known to affect vasovagal response. However, their specific roles are unclear ${ }^{3,4}$. Thus the rationale of this study was to ascertain whether either one or both peptides are substantially involved in the course of VVS. Galanin (GAL) is a 30 -amino acid peptide with almost ubiquitous presence in organisms within the central and peripheral nervous system, especially cardiac sympathetic neurons ${ }^{5}$. Its amino acid sequence is conserved from vertebrates to mammals which indicates its possible importance ${ }^{6}$. GAL has been proposed to take part in the regulation of cardiovascular homeostasis ${ }^{7}$, more precisely GAL was demonstrated to lower norepinephrine plasma levels and thus attenuate blood pressure response to orthostasis ${ }^{8}$. Further principal actions of GAL are inhibition of acetylcholine, glutamate, and insulin release, stimulation of feeding, stimulation of pituitary hormone release, and inhibition of spinal nociceptive reflexes ${ }^{9}$. The 52-amino acid adrenomedullin $(\mathrm{ADM})$ is a relatively strong vasorelaxant and natriuretic peptide ${ }^{10}$. Like GAL, ADM has a very conserved amino acid sequence which places ADM in the evolutionary line at the pre-dinosaur age ${ }^{11}$. For this reason, ADM has been studied in many disease states including hypertension, heart and renal failure. ADM is constitutively produced by endothelial cells and regulated on the level of gene 
expression $^{12}$. It has been reported that the ADM plasma levels increase during the head-up tilt test (HUTT) - induced passive orthostasis - followed by a decrease after supination ${ }^{4}$. In the present study, we investigated whether the secretion patterns of GAL and ADM during HUTT differ in patients suffering from recurrent vasovagal syncope (rVVS) and for healthy individuals. We concentrated both on the total level of blood plasma GAL and ADM (absolute values) and on their rates of change (relative values). We also investigated whether the occurrence of a syncope during HUTT affected the secretion patterns. Vagal tone is known to increase during typical vasovagal syncope. Thus we employed the brake index as an indirect measure of vagal influence.

\section{MATERIALS AND METHODS}

\section{Subjects}

14 patients ( 7 men, 7 women) with recurrent vasovagal syncope (rVVS), without structural heart or neurological diseases were enrolled as cases. For the purpose of the study we defined rVVS as a history of one or more syncopes and at least one syncope reproducible during HUTT. All cases experienced 2 or more syncopes per month and all had had vasodepressor type of vasovagal syncope during HUTT prior to enrollment. Another 14 healthy individuals (11 women, 3 men, all medical staff volunteers) without a history of syncopes or presyncopal states were used as controls. Absence of structural heart disease, absence of medication, normal ECG and physical status including neurotopic state were the principal inclusion criteria. The BMI of cases was $23.55 \pm 3.24$ and controls $22.97 \pm 2.59$.

\section{Study protocol}

Both groups were required to refrain from consuming tea, coffee, chocolate and smoking cigarettes two days before the testing. HUTT was performed between 8:30 and 11:00 AM in a quiet, well ventilated room after overnight fasting and all subjects were well hydrated. Before the HUTT started, antecubital vein cannulation was undertaken for further blood sampling. All patients were asked to rest for $10-15 \mathrm{~min}$ in a supine position before the test to allow all the cardiovascular parameters (pulse, blood pressure, respiration rate) to stabilize. The HUTT then proceeded according to the shortened protocol to eliminate late false positive results: the subjects were tilted at $60^{\circ}$ head upwards, with their feet secured, for $35 \mathrm{~min}$, without medical provocation. All subjects were further stratified according to HUTT result. Blood samples were taken in the first minute of the test and then, if syncope developed (positive HUTT), in the first 10-15 s and tenth min. afterwards. After the syncope developed, blood samples were first taken and then the patients were returned to the supine position. If the HUTT was negative (no syncope occurred), blood samples were taken after 1, 25, and 35 min of the test. Blood pressure was measured every two min and then every minute in the presyncopal phase and
ECG was recorded continuously for further heart rate variability analysis (brake index). Both blood pressure and ECG were recorded by the Datex Ohmeda S5 monitoring system. ECG recordings were used for brake index calculation (RRmax-RRmin/RRresting), which is a simple heart rate variability measure used to assess the amount of vagal influence. The test procedure was attended by a nurse and a physician. The study protocol was approved by the Local Ethics Committee of the University Hospital in Ostrava. The trial was carried out in accordance with the principles of the Helsinki Declaration. Written informed consent was obtained from all patients prior to enrollment.

\section{Analytical methods}

Blood samples were drawn into pre-cooled tubes containing EDTA and aprotinin (Within $30 \mathrm{~min}$, the samples were centrifuged at $1600 \mathrm{~g} / 4{ }^{\circ} \mathrm{C}$ for $15 \mathrm{~min}$, and then the plasma was frozen at $-80{ }^{\circ} \mathrm{C}$ until the analysis which was performed within four months. Plasma ADM levels were measured by commercially available ELISA kit for human ADM containing 52 amino acids (EK-010-01, Phoenix Pharmaceuticals, for research only use). The detection range is $0.13-100 \mathrm{ng} / \mathrm{mL}$. Plasma GAL levels were obtained by means of commercially available RIA kit (RK-026-01, Phoenix Pharmaceuticals, for research only use). The detection range is $10-1280 \mathrm{pg} / \mathrm{mL}$.

\section{Statistical methods}

Unless stated otherwise, median values of ADM and GAL plasma levels are provided as non-parametric statistical tests were generally used (the hypothesis of normality of the data was rejected, $P<0.0001)$. All analyses of ADM and GAL levels (including their rates of change) were performed by the Kruskal-Wallis test. Break index (see the results below) data were analysed using ANOVA or t-tests as the data were normally distributed. P values below 0.05 were considered statistically significant.

\section{RESULTS AND DISCUSSION}

A HUTT induced syncope occurred in 4/14 healthy individuals after $3,26,27$, and $30 \mathrm{~min}$ of the test. In the rVVS patients, 8/14 individuals suffered a vasovagal syncope after 4, 11, 21, 22, 24, 24, 26, and $27 \mathrm{~min}$. Although the time to syncope was different in the rVVS group, we believe the pathophysiological mechanisms are uniform. Moreover by holding the blood sampling time frame possible differences are diminished. Basic clinical characteristics and haemodynamic parameters of the groups are shown in (Table 1). First, we compared the plasma levels of ADM and GAL for positive cases and negative controls (where the most profound difference is to be expected) for all three measurements (see the study protocol). No significant difference between the groups was found ( $P=0.92$ and $P=0.98$ ). Next, to capture the dynamics of the regulatory system, we compared the rates of change of the ADM and GAL levels between consecutive measurements (i.e. the change in concentration divided by time). The rate of 
Table 1. Basic characteristics of the study group. All values are provided as the mean \pm standard deviation. Normality of the data was not rejected.

\begin{tabular}{|c|c|c|c|c|c|c|c|}
\hline & \multicolumn{3}{|c|}{ Healthy subjects (controls) } & \multicolumn{3}{|c|}{ rVVS patients (cases) } \\
\hline & & $\begin{array}{l}\text { HUTT negative } \\
\quad(\mathrm{n}=10)\end{array}$ & $\begin{array}{l}\text { HUTT positive } \\
\quad(\mathrm{n}=4)\end{array}$ & $P$ & $\begin{array}{l}\text { HUTT negative } \\
\quad(\mathrm{n}=6)\end{array}$ & $\begin{array}{l}\text { HUTT positive } \\
\quad(\mathrm{n}=8)\end{array}$ & $P$ \\
\hline \multicolumn{2}{|l|}{ Age (years) } & $24.5 \pm 2.5$ & $26.3 \pm 4.3$ & 0.48 & $41.0 \pm 16.0$ & $34.1 \pm 12.6$ & 0.36 \\
\hline \multicolumn{2}{|l|}{ Sex } & $9 \mathrm{~F} / 1 \mathrm{M}$ & $1 \mathrm{~F} / 3 \mathrm{M}$ & & $2 \mathrm{~F} / 4 \mathrm{M}$ & $4 \mathrm{~F} / 4 \mathrm{M}$ & \\
\hline \multicolumn{2}{|l|}{ BMI $\left(\mathrm{kg} / \mathrm{m}^{2}\right)$} & $23.1 \pm 2.5$ & $22.6 \pm 3.1$ & 0.72 & $22.7 \pm 3.4$ & $23.1 \pm 2.7$ & 0.95 \\
\hline \multirow{2}{*}{$\begin{array}{l}\text { Systolic BP } \\
(\mathrm{mmHg})\end{array}$} & Supine & $114.7 \pm 8.1$ & $122.5 \pm 11.9$ & 0.20 & $127.8 \pm 10.2$ & $124.0 \pm 16.3$ & 0.30 \\
\hline & Tilting ( $3 \mathrm{~min})$ & $115.7 \pm 9.7$ & $112.3 \pm 15.7$ & 0.72 & $131.7 \pm 10.7$ & $119.8 \pm 26.2$ & 0.03 \\
\hline \multirow{2}{*}{$\begin{array}{l}\text { Diastolic BP } \\
(\mathrm{mmHg})\end{array}$} & Supine & $69.4 \pm 5.7$ & $77.8 \pm 5.2$ & 0.02 & $82.8 \pm 6.9$ & $76.5 \pm 7.2$ & 0.10 \\
\hline & Tilting ( $3 \mathrm{~min})$ & $74.5 \pm 5.3$ & $73.8 \pm 14.9$ & 0.94 & $86.8 \pm 11.9$ & $75.4 \pm 15.0$ & 0.10 \\
\hline \multirow{2}{*}{$\begin{array}{l}\text { Heart rate } \\
\text { (beats/min) }\end{array}$} & Supine & $62.3 \pm 5.1$ & $67.0 \pm 10.4$ & 0.44 & $61.2 \pm 6.8$ & $59.6 \pm 12.7$ & 0.79 \\
\hline & Tilting (3min) & $74.5 \pm 7.9$ & $77.0 \pm 11.2$ & 0.62 & $74.2 \pm 10.6$ & $82.0 \pm 19.0$ & 0.44 \\
\hline
\end{tabular}
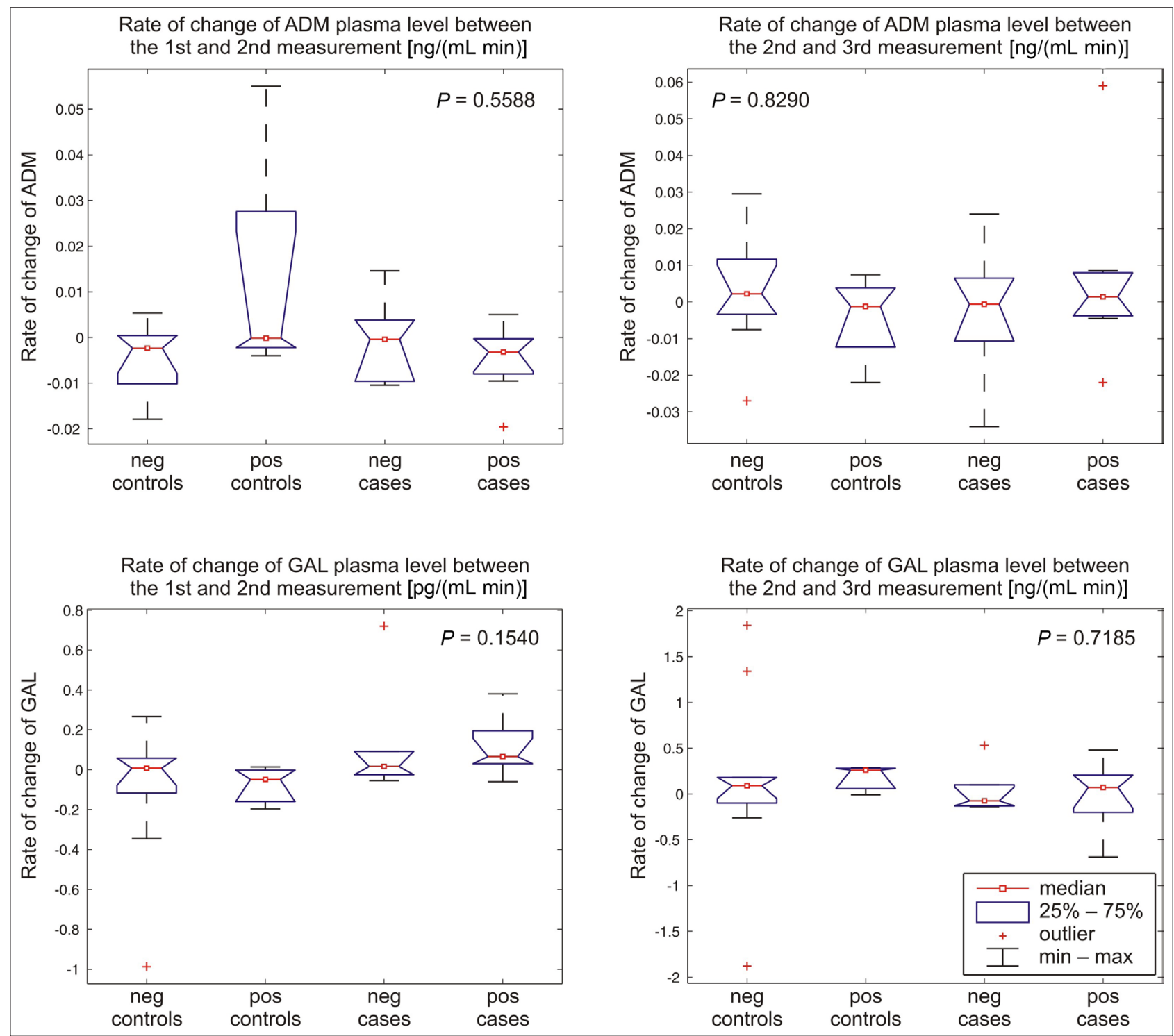

Fig. 1. Rate of change of Adrenomedullin and Galanin plasma level for all four subgroups between the $1^{\text {st }}$ and $2^{\text {nd }}, 2^{\text {nd }}$ and $3^{\text {rd }}$ measurement, respectively. 


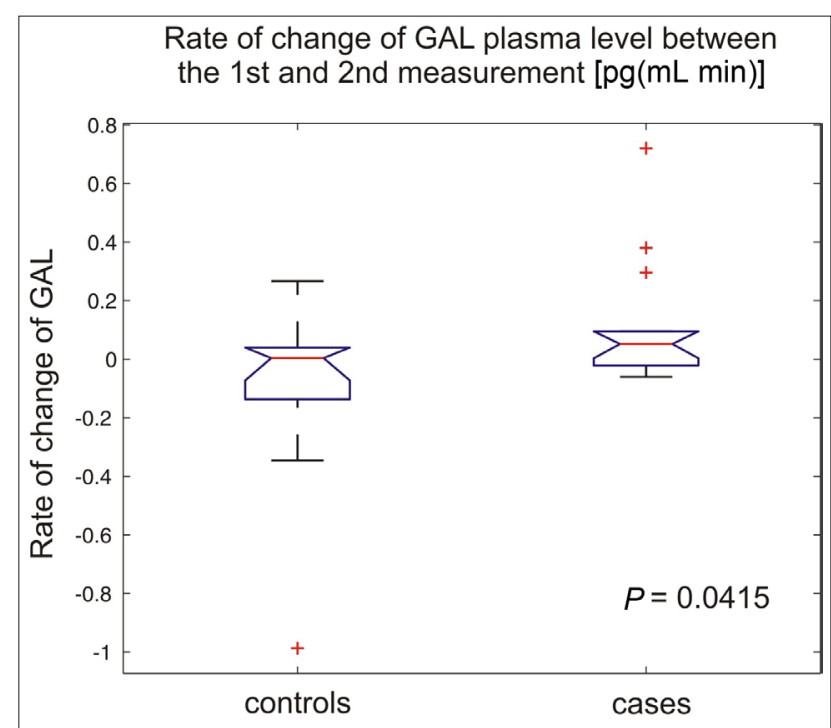

Fig. 2. Rate of change of Galanin between the $1^{\text {st }}$ and $2^{\text {nd }}$ measurement, cases and controls, independent on the result of the HUTT.

change of ADM plasma level between the first and second measurement was not significantly different for any of the four subgroups (positive cases, negative cases, positive controls, negative controls, $P=0.56$ ). Although the box plot (Fig. 1) shows a mild increase in ADM for positive controls, by contrast the remaining three groups show stagnation or slight decrease. The non-significance of this difference may be caused by the small study group. There was also no significant difference in ADM plasma level changes between the second and third measurement: the levels of ADM tend to stagnate for all the four subgroups (Fig. 1). The same analysis was applied to GAL levels. The variation of GAL plasma level change between the first and second measurement is insignificant (Fig. 1), however for the controls, GAL tends to decrease, while for cases there is an opposite tendency. Comparing cases and controls with no further differentiation into positive and negative groups, this difference becomes significant ( $P=0.04$, Fig. 2). Patients suffering from rVVS (cases) experience an increase in GAL levels, while healthy individuals experience a decrease, regardless of the HUTT result. The variation in GAL plasma level change between the second and third measurement shows no significant difference between the four groups $(P=0.72$, Fig. 1$)$, not even when only cases and controls are compared (without differentiating positive and negative). We further tried to capture the secretion patterns for GAL and ADM qualitatively, assigning to each patient one of the three secretion types: an "A-type" for an increase between the first two measurements followed by a decrease, a "V-type" for a decrease followed by an increase, and an "N-type" for any other. This type of analysis showed no significant differences among the groups or subgroups, for either ADM or GAL levels. It is known that the so-called break index ${ }^{13}$, is a good marker of elevated vagal influence. Accordingly, in the positive cases, the values of the break index increased significantly ( $P=0.02$, Fig. 3 ). It is also noteworthy that

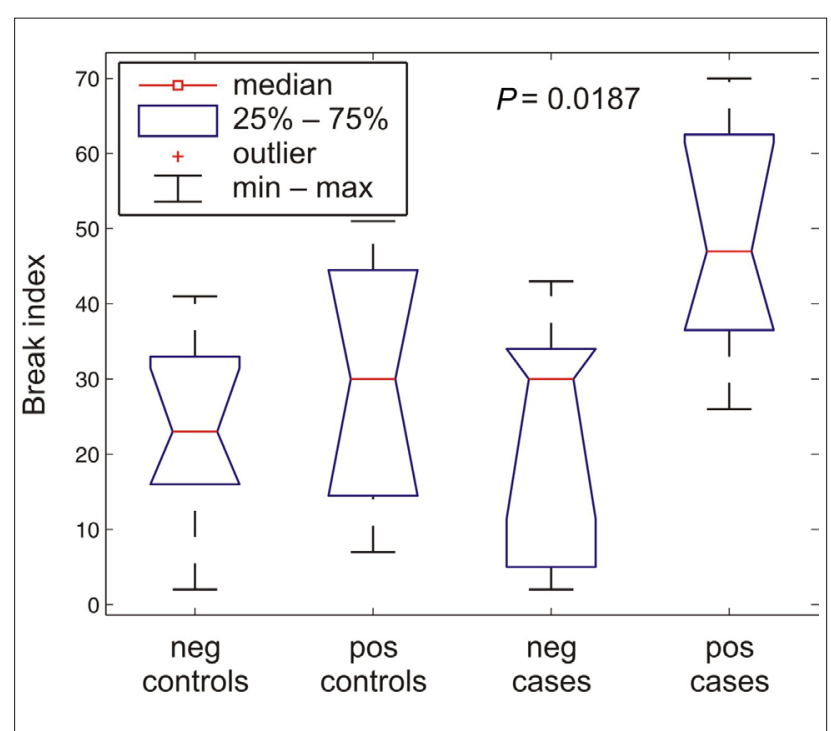

Fig. 3. Brake index, all four groups (positive and negative control, positive and negative cases).

there is a significant correlation $(\mathrm{r}=0.48, P=0.01)$ between the range of $\mathrm{ADM}$ levels (i.e. the maximum level minus the minimum level) and the range of GAL levels in the group of all patients.

As stated a number of neurohumoral factors are involved in triggering and sustaining the vasovagal response. However, neither the roles of individual peptides nor their integrated response is known. In this study, the role of $\mathrm{ADM}$ and GAL was addressed to a small group of patients with rVVS. The secretion patterns of both peptides during HUTT were compared to a group of healthy individuals. The authors are not aware of any other HUTT trial without pharmacological provocation performed on rVVS patients while measuring ADM levels though a case/control study exists ${ }^{14}$ evaluating GAL levels in patients with rVVS during HUTT. In another trial ${ }^{15}$, adrenomedullin response to HUTT in VVS patients was assessed. However, in most patients, the syncope was induced by nitroglycerin administration, and the condition of syncope recurrence was not satisfied. Bondanelli et al. ${ }^{14}$ show that GAL levels increase in rVVS patients who do not develop a syncope (negative HUTT), whereas it remains unchanged in rVVS patients who develop a syncope (positive HUTT). GAL levels are reported to remain unchanged in the control group but there were no positive responses to the HUTT in this group $^{14}$. This suggests that GAL could be responsible for preventing a syncope development in susceptible individuals. We observed more stereotypical GAL response to tilting in all subjects. In our trial, GAL increase differentiated cases from controls better than it differentiated positive and negative responses to HUTT, i.e. there was an increase in plasma GAL for rVVS patients during HUTT, regardless whether they developed syncope or not. Our data are thus in a partial conflict with those of Bondanelli et al. Elsewhere it has been demonstrated in healthy individuals that GAL plasma levels increase severalfold with orthostatic stress in the presyncopal phase ${ }^{3}$, which further 
questions the reproducibility of the results of Bondanelli. A possible explanation for this conflict may be the absence of a unique GAL secretion pattern. In other words, GAL response to orthostasis might be more complex than its mere increase/decrease, considering the number of other neurohumoral agents involved ${ }^{16}$. Our results show that there is no strong adherence of galanin secretion pattern to orthostatic stimuli, although the galaninergic system is involved in the vasovagal response. The galanin secretion pattern better differentiates patients with rVVS from controls than the type of response to HUTT (positive/negative). This poses the question whether rVVS is just an abnormal autonomic/neurohumoral reaction or a genuine disease ${ }^{17}$.

No significant change in ADM plasma levels was observed in our trial, although there was a slight increase in ADM plasma levels in healthy individuals with a positive response to HUTT. It was shown that ADM plasma levels increased significantly with tilting and this correlated with the tilt table inclination level in healthy individuals ${ }^{4}$. Similar results were reported by Gajek et al. ${ }^{15}$, who demonstrated increased ADM plasma levels during HUTT immediately after syncope in patients with vasovagal vasodepresor reaction. The opposite was true (i.e. the ADM levels decreased) if nitroglycerin was administered ${ }^{15}$. This advocates the role of ADM in a failed prevention of a syncope by excessive paradoxical vasodilatation in healthy individuals and the supression of this effect by nitroglycerin administration. Our results show an increasing trend in ADM levels in healthy individuals during HUTT which partly supports Rossler et al. ${ }^{4}$. Although a different protocol and tilt angle were used Nishikimi et al. ${ }^{18}$ failed to demonstrate ADM increase after $20 \mathrm{~min}$ of $70^{\circ} \mathrm{HUTT}$ in healthy volunteers. The brake index is used as a measure of the activity of the autonomic nervous system as a whole ${ }^{13,19}$. The significantly elevated brake index for rVVS patients with a positive HUTT result compared with healthy individuals shows that the rVVS patients were well-chosen: increased heart rate variability determinated by the brake index is a sign of increased vagal influence.

\section{CONCLUSION}

This study confirms that the galaninergic system is involved in the reaction of rVVS patients to HUTT. It also suggests that GAL accruement throughout HUTT might be used as a marker of vasovagal syncope recurrence. It also poses the question whether rVVS represents an abnormal response to orthostasis/HUTT as a variant of a normal reaction or a genuine disease. To our knowledge, this study represents the first evaluation of ADM in heavily symptomatic rVVS patients during a HUTT which was not accompanied by drug administration. No specific ADM secretion pattern was found for patients with rVVS and our patient's ADM plasma levels were generally lower than the controls. This may be due to fast biodegradation or re-uptake of ADM by the endothelial cells. The significant correlation of GAL and ADM ranges suggests that both peptides are part of the same regulatory pathway, although possibly acting in mutual opposition. The results do not answer all the questions on the involvement of both vasoactive peptides in rVVS and for this reason further research on neurohumoral response to syncope is needed to enhance understanding of this sophisticated system.

Small sample size is the major limitation of the study, however, most of the trials cited in this paper have similar numbers of patients enrolled. Cases and controls are gender unbalanced, which might affect especially the GAL plasma levels. It was shown that galanin plasma levels during orthostasis are higher in healthy women (3), however, our data do not confirm this.

\section{AKNOWLEDGEMENT}

This work was financially supported by AstraZeneca Czech Republic s.r.o.

\section{CONFLICT OF INTEREST STATEMENT}

Author's conflict of interest disclosure: The authors stated that there are no conflicts of interest regarding the publication of this article.

\section{REFERENCES}

1. The European Society of Cardiology Guidelines for the diagnosis and management of syncope reviewed by Angel Moya, MD, FESC, Chair of the Guideline Taskforce with J. Taylor, MPhil. Eur Heart J 2009;30:2631-71.

2. Mosqueda-Garcia R, Furlan R, Tank J, Fernandez-Violante R. The elusive patho-physiology of neurally mediated syncope. Circulation 2000;102:2898-2906.

3. Hinghofer-Szalkay HG, Rössler A, Evans JM, Stenger MB, Moore FB, Knapp CF. Circulatory galanin levels increase severalfold with intense orthostatic challenge in healthy humans. J Appl Physiol 2006;100(3):844-9.

4. Rössler A, Lászlo Z, Haditsch B, Hinghofer-Szalkay HG. Orthostatic stimuli rapidly change plasma adrenomedullin in humans. Hypertension 1999: 34(5):1147-51.

5. Vrontakis ME. Galanin: A Biologically Active Peptide. Curr Drug Targets CNS Neurol Disord 2002;1(6):531-41.

6. Wynick D, Bacon A. Targeted disruption of galanin: new insights from knock-out studies. Neuropeptides 2002;36(2-3):132-44.

7. Diaz-Cabiale Z, Narvaez JA, Yanaihara N, Gonzales-Baron SM, Fuxe K. Galanin/alpha2-receptor interactions in central cardiovascular control. Neuropharmacology 2000;39(8):1377-85.

8. Degli Uberti EC, Ambrosio MR, Bondanelli M, Trasfori G, Margutti A, Valentini A, Rosi R, Franceschetti P. Human galanin reduces plasma norepinephrine levels in man. J Clin Endocrinol Metab 1995;80(6):1894-8.

9. Crawley JN. Biological actions of galanin. Regul Pept 1995;59(1):1-16.

10. Brain SD, Grant AD. Vascular actions of calcitonin gene-related peptide and adrenomedullin. Physiol Rev 2004;84(3):903-34.

11. Martinez A, Cuttita F. Adrenomedullin. Amsterdam: IOS press;1998.

12. Sugo S, Minamino N, Kangawa K, Miyamoto K, Kitamura K, Sakata J, Eto T, Matsuo H. Endothelial cells actively synthesize and secrete adrenomedullin. Biochem Biophys Res Commun 1994; 201:1160-6.

13. Cybulski G, Niewiadomski W. Influence of age on the immediate heart rate response to the active orthostatic test. J Physiol Pharmacol 2003;54(1):65-80. 
14. Bondanelli $M$, Alboni $P$, Margutti A, Franceschetti $P$, Dinelli $M$, Gruppilo P, Marchi P, Degli- Uberti EC. Plasma galanin response to head-up tilt in normal subjects and patients with recurrent vasovagal syncope. Metabolism 2003;52(3):315-21.

15. Gajek J, Zyśko D, Halawa B. Adrenomedullin-the link between the sympathetic nervous system activation and peripheral vasodilatation in some patients with vasovagal syncope. Pol Merkur Lekarski 2004; 17(99):267-70.

16. Sander-Jensen K, Secher NH, Astrup A, Christensen NJ, Giese J, Swarz TW, Warberg J, Bie P. Hypotension induced by passive head-up tilt: endocrine and circulatory mechanism. Am J Physiol 1986;251:74248.

17. Alboni $\mathrm{P}$, Brignole $\mathrm{M}$, Degli Uberti EC. Is vasovagal syncope a disease? Europace 2007;9(2):83-7.

18. Nishikimi T, Junichi M, Yasu T, Takeshi S, Kangawa K, Matsuoka H. Two molecular forms of plasma adrenomedullin during tilt test in healthy subjects. Peptides 2001;22(11):1867-72.

19. Wieling W, Borst C, Karemaker JM, Dunning AJ. Testing for autonomic neuropathy: initial heart rate response to active and passive changes of posture. Clin Physiol 1985;5:23-7. 